\title{
Les systèmes spécifiques
}

L'appellation de "systèmes spécifiques " s'applique aux systèmes qui assurent les grandes fonctions sensorielles et motrices du système nerveux central et présentent certaines propriétés particulières communes d'organisation anatomique et fonctionnelle. De nombreux systèmes peuvent être considérés comme "spécifiques ", tant sur le versant sensoriel (système auditif, visuel, somesthésique, gustatif, le système de la sensibilité profonde proprioceptive, les systèmes liant la moelle épinière au cervelet directement ou après relais dans l'olive inférieure) que sur le versant effecteur (principales composantes de la commande motrice telles que les systèmes reliant le cortex cérébral ou le noyau rouge à la moelle épinière, ou les voies indirectes associant le cortex cérébral et le cervelet). Parmi cet ensemble, les systèmes somesthésique et corticospinal peuvent être considérés comme des "prototypes" (figure 1).

La qualification de "spécifique " est due aux propriétés fonctionnelles de ces systèmes. En effet, sur le versant afférent, les récepteurs périphériques, en fonction des caractéristiques de nature, durée et intensité des stimuli qui sont capables de les activer avec une grande spécificité, jouent un rôle de transducteurs et engendrent des trains de potentiels d'action - ou messages afférents - codant en fré- ristiques de ces stimuli. Ensuite, à chaque relais dans la chaîne neuronale, les fonctions de codage sont respectées et la signification des messages est conservée. En fait, la signification des messages, à la fois qualitative et quantitative, serait perdue si ceux-ci aboutissaient à un réseau nerveux de type convergent comme celui de systèmes non spécifiques $(\mathrm{m} / \mathrm{s}$ $n^{\circ}$ 6, vol. 6, p. 580).

Il n'en est rien puisque l'on peut montrer que chacun de ces systèmes présente des connexions précises où les communications entre relais contribuent à la constitution de cartographies ("topies") dépendant d'un ou plusieurs, paramètres du stimulus d'origine. Par exemple, au niveau du système visuel ou du système somatosensoriel, on peut décrire des champs récepteurs dès les cellules rétiniennes ou de la corne dorsale de la moelle ; ceux-ci correspondent aux surfaces (rétiniennes ou cutanées) bien délimitées d'où proviennent les messages afférents aux cellules chargées ensuite de les convoyer vers le relais suivant. Ainsi, à chacune des étapes suivantes, par exemple dans les noyaux thalamiques puis au niveau des projections corticales primaires, on retrouvera cette organisation "point par point " - ou topique - reproduisant au niveau central la surface rétinienne ou corporelle (figure 1).

Cette organisation à la fois linéaire et en parallèle des voies de circula- tion des messages repose sur deux propriétés des systèmes : l'une, anatomique, est liée au "câblage " en parallèle des lignes neuronales véhiculant de la périphérie jusqu'aux centres les messages issus de champs récepteurs contigus. L'autre, fonctionnelle, repose sur les interactions dynamiques entre les voies parallèles décrites ci-dessus, par des phénomènes d' "inhibition latérale ". Ceux-ci siègent au niveau des relais successifs et permettent à un message particulier d'être fortement contrasté par rapport au bruit de fond de l'activité sensorielle globale. Le message conserve ainsi ses qualités informatives sur la nature, l'intensité et la durée de la stimulation qui lui a donné naissance.

Cette spécificité dans l'organisation des voies de communication entre les différents centres impliqués dans une même fonction se retrouve également pour les systèmes efférents de la commande motrice et est particulièrement bien illustrée par l'" homunculus" que l'on peut dessiner sur la convexité du cortex moteur primaire (figure 1): la stimulation ponctuelle et liminaire de l'une de ces zones corticales délimitées de manière très précise provoquera la mobilisation d'un territoire musculaire correspondant qui pourra se limiter à une seule unité motrice.

Enfin, l'organisation des systèmes spécifiques repose sur certaines pro- 


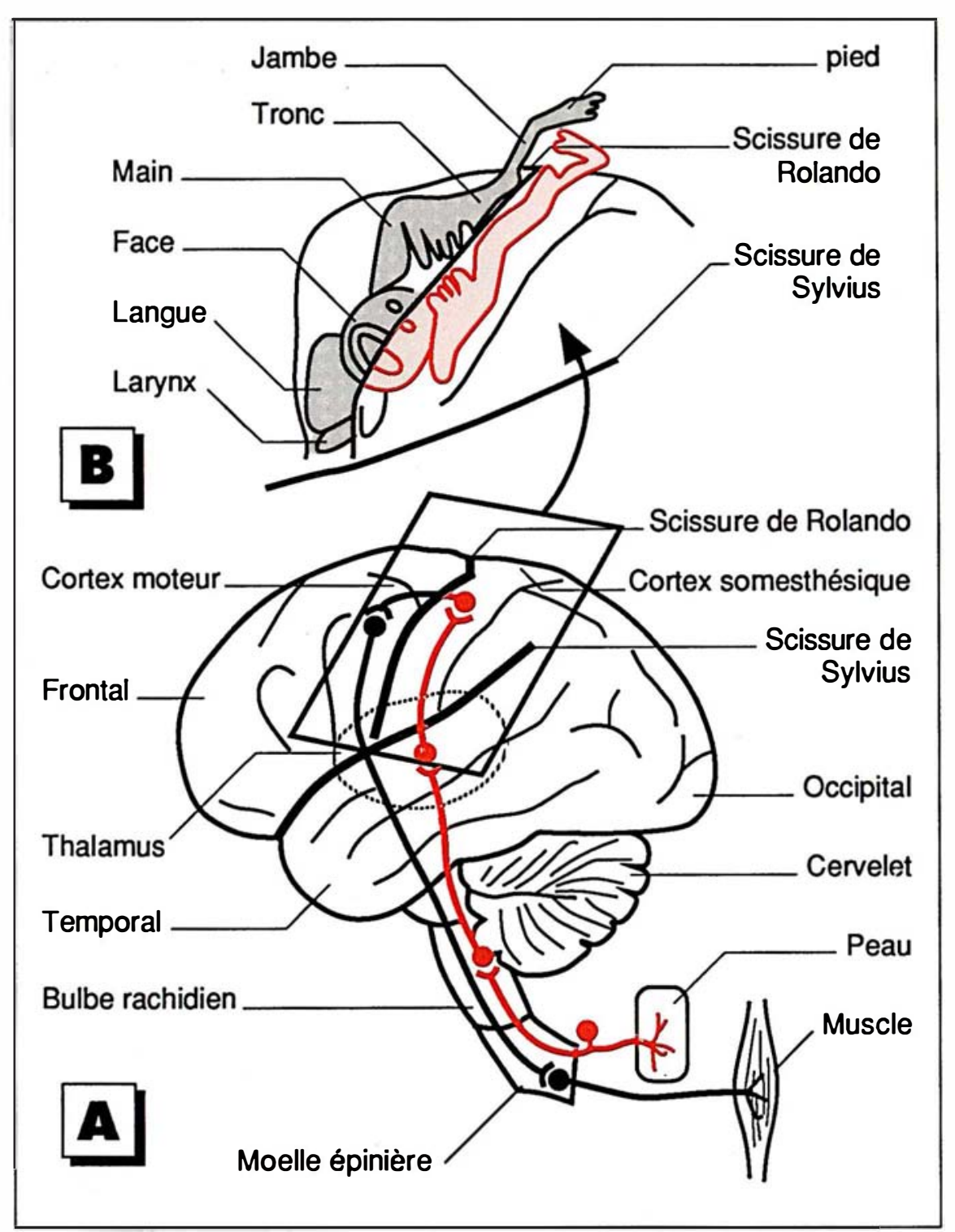

Figure 1. Représentation schématique des systèmes somesthésique (rouge) et cortico-spinal (noir); à partir des récepteurs cutanés, le système afférent est construit "linéairement", de même pour le versant efférent moteur ; la partie $B$ est un grossissement de la fenêtre de la partie $A$ dans laquelle on a dessiné les "homunculus ": les topies spécifiques sont schématiquement reproduites de part et d'autre de la scissure de Rolando, en prérolandique, la topie motrice homunculus moteur), en post-rolandique, la somatotopie somatosensorielle. Le dessin indique les surfaces à partir desquelles on peut recueillir les messages somatiques (aire somesthésique) ou celles dont la stimulation déclenche une activité musculaire isolée (aire motrice). On peut noter la taille irrégulière de la représentation corticale des régions du corps, qui est proportionnelle, soit à la densité de l'innervation sensorielle cutanée, soit à l'étendue du domaine musculaire contrôlé.

$\mathrm{m} / \mathrm{s} n^{\circ} 7 \mathrm{vol} .6$, septembre 90 priétés d'organisation synaptique. En premier lieu, le nombre de neurones composant un circuit élémentaire est faible: trois neurones dans le système somesthésique et le système visuel entre l'organe des sens et le cortex cérébral ; deux neurones pour la commande motrice cortico-spinale. En deuxième lieu, ces circuits de base étant simples, les jonctions synaptiques y sont peu nombreuses et peu fatigables, admettant des fréquences élevées de transmission des messages, avec une fiabilité importante et peu de possibilités de brouillage. La transmission des informations dans de tels systèmes se fera donc avec un très fort coefficient d'efficacité et de sécurité d'un bout à l'autre de la chaîne.

De plus, ces propriétés synaptiques pourraient être reliées au fait que, souvent, les neurotransmetteurs utilisés seraient des petites molécules de la famille des acides aminés excitateurs ou inhibiteurs qui permettraient des fonctionnements synaptiques phasiques et répétitifs, ce qui ne serait probablement pas le cas d'une neuromédiation peptidergique.

En conclusion, on peut dire que les systèmes spécifiques sont constitués par des récepteurs et des effecteurs spécialisés associés à des voies d'entrée et sortie du système nerveux par lesquelles transitent des messages ayant un contenu informatif très spécialisé. Cette organisation permet au système nerveux central de recevoir des informations à tout moment sur l'état de l'organisme et de son environnement et d'organiser par des ajustements comportementaux notamment moteurs - des réponses rapides et adaptées.
Jean-Paul Rivot Bernard Calvino Marc Peschanski
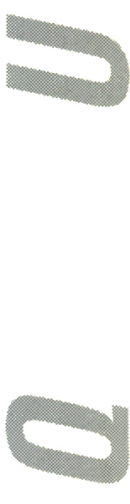

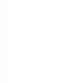

